\title{
Peak-to-Average Power Ratio Analysis in Multicode CDMA
}

\author{
K.Sathananthan* and C. Tellambura ${ }^{\dagger}$ \\ *School of Computer Science and Software Engineering, Monash University \\ Clayton, Vic 3168, Australia. Email: satha@csse.monash.edu.au \\ ${ }^{\dagger}$ Department of Electrical and Computer Engineering, University of Alberta \\ Edmonton, Alberta, Canada. Email: chintha@ee.ualberta.ca
}

\begin{abstract}
This paper analyzes the Peak-to-Average Power Ratio (PAPR) problem in Multicode-Code Division Multiple Access (MC-CDMA) systems. The statistical distribution of PAPR is derived and the achievable PAPR reduction for a given code rate is estimated. We show that the PAPR in MC-CDMA communications systems can be reduced by Partial Transmit Sequence (PTS) and Selected Mapping (SLM) approaches. In PTS, the subblocks are multiplied by a set of phase factors that are optimized to minimize PAPR. In SLM, several independent data frames are generated and the data frame with the lowest PAPR is selected for transmission. We also show that BER performance improves when the PAPR-reduced MC-CDMA signal is passed through a nonlinear amplifier. SLM reduces the error floor caused by amplifier nonlinearities and offers an SNR gain of $6 \mathrm{~dB}$ at $10^{-5}$ BER with an input back-off of $1 \mathrm{~dB}$.
\end{abstract}

\section{INTRODUCTION}

Third generation (3G) wireless systems aim of providing universal access and global roaming. Introduction of wideband packet data services for wireless Internet up to $2 \mathrm{Mb} / \mathrm{s}$ will probably be the main attribute of $3 \mathrm{G}$ systems [1-3]. Multicode CDMA has been proposed for such systems [4]. Unlike classical CDMA, a Walsh-Hadamard transform (WHT) of input data is taken before spreading in MC-CDMA. This allows rate adaptation. However, the WHT output is not a binary signal, rather a multilevel signal. Thus a multicode signal can have a large variation in the envelope and a nonlinearity can cause a problem. The efficiency of a high power amplifier (HPA) is limited due to high peak-to-average power ratio (PAPR).

Since this problem is clearly parallel to that of OFDM, it is worthwhile to mention that the block coding approach for PAPR reduction in OFDM was first proposed in $[5,6]$. The basic idea is to not transmit OFDM symbols with large PAPR values. This requires that both the transmitter and the receiver keep a list of "allowed" data frames (ie., a codebook). Unfortunately, this implementation fails for large $n$, where $n$ is the size of the WHT. However, this failure motivates algebraic encoding/decoding for such block codes. To this end, progress has been made on several fronts. First, PAPR reduction codes using Golay complementary sequences and second-order Reed-Muller codes have been developed $[7,8]$. This method ensures PAPR at most $3 \mathrm{~dB}$ while allowing simple encoding and decoding for binary, quaternary or higher-phase signalling together with good error correction. However, these codes suffer from vanishing code rate as $n$ increases.

Similarly, for MC-CDMA several PAPR reduction schemes have been proposed and investigated. In [9-11] precoding techniques to reduce the signal envelope variations are developed, with precoder designs based on simulated and analytical expressions. Constant amplitude coding techniques are also developed in [12-15], but these are ad-hoc and valid for short lengths only. In $[16,17]$ the impact of an amplifier nonlinearity is investigated for various multicode systems. In $[18,19]$, PAPR reduction codes are systematically studied. Codes which are distant(Hamming sense) from the first-order Reed-Muller code will have small PAPR. Bounds on the trade-off between rate, PAPR and error-correcting capability of codes for MC-CDMA are also derived. The connections between the code design problem, bent functions and algebraic coding theory are exploited to construct code families.

We add to the above contributions by proposing the use of PTS [20,21] and SLM [20] to reduce the PAPR in MCCDMA systems. Generating several statistically independent OFDM frames for a data frame and selecting the one with the lowest PAPR is a common approach in [20,21]. This approach improves the statistics of the PAPR at the expense of additional complexity. However, an advantage is that this approach does not require much redundancy compared to that of coding. That is to say, PAPR-reduced coding can lead to very low code rates.

\section{PAPR OF MULTICODE-CDMA}

\section{A. PAPR definition}

The output of the WHT can be represented as

$$
\mathbf{S}=H_{n}\left(c_{0}, c_{1}, \ldots, c_{n-1}\right)^{T}
$$

where $c_{k} \in\{+1,-1\}$ is a data symbol. The $n \times n$ Hadamard matrix $H_{n}$ is a matrix whose entries are limited to \pm 1 and can be defined recursively by $H_{1}=(1)$ and

$$
H_{n}=\left(\begin{array}{cc}
H_{n / 2} & H_{n / 2} \\
H_{n / 2} & -H_{n / 2}
\end{array}\right)
$$

We shall write ordered $n$-tuples $\mathbf{c}=\left(c_{0}, c_{1}, \ldots, c_{n-1}\right)$ and $\mathbf{S}=\left(S_{0}, S_{1}, \ldots, S_{n-1}\right)$. The sequence $\left\{S_{k}\right\}$ is a multilevel 
sequence, with levels varying from $-n$ to $+n$. The transmitted signal is obtained by multipling $S_{k}$ by a spreading sequence, filtering and frequency up conversion. Details of these operations are omitted, as they do not greatly affect the PAPR. A simplified block diagram of the transmitter in MC-CDMA system is shown in Fig. 1.

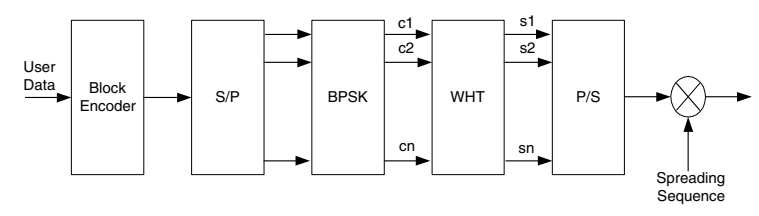

Fig. 1. Simplified Block Diagram of the Transmitter in MC-CDMA System

The peak-to-average-power ratio (PAPR) is defined as

$$
\operatorname{PAPR}(\mathbf{c})=\frac{1}{n} \max _{0 \leq k \leq n-1}\left|S_{k}\right|^{2}
$$

The PAPR is a function of the data frame and there are $2^{n}$ distinct data frames. For any input data frame, we have

$$
1<\operatorname{PAPR}(\mathbf{c}) \leq \mathbf{n}
$$

For example, for $n=256$ the PAPR can be as high as 24 $\mathrm{dB}\left(10 \log _{10}(256)\right)$. Very high PAPR values are very rare though. If $n=2^{m}$, all sequences that achieve $\operatorname{PAPR}(\mathbf{c})=$ $\mathbf{n}$ must be codewords of $\operatorname{RM}(1, m)$ (first order Reed-Muller code of length $2^{m}$ ). There are $2^{m+1}$ such codewords. For randomly distributed data, the probability of an occurrence of the highest peak is $2^{m+1} / 2^{n}=2^{m+1-n}$. This probability is negligible when $n$ is large, as is the case in practice.

\section{B. CCDF of the PAPR}

Since the input data is randomly distributed in many applications (if not, it can be made so by the use of a suitable scrambling operation), $\operatorname{PAPR}(\mathbf{c})$ itself is a random variable. The complementary cumulative distribution function (CCDF), the probability that the PAPR of a MC-CDMA symbol exceeds a certain threshold, is useful for many purposes. The CCDF is defined as

$$
F_{c}(\zeta)=\operatorname{Pr}(\operatorname{PAPR}(\mathbf{c}) \geq \zeta)
$$

In order to determine this distribution, we first evalulate the statistical distribution of $S_{k}$. The data symbols $c_{k}$ are discrete random variables with $\operatorname{Pr}\left(c_{k}=1\right)=$ $\operatorname{Pr}\left(c_{k}=-1\right)=\frac{1}{2}$. Since each row of $H_{n}$ consists of +1 and $-1, S_{k}$ can be modeled by the binomial distribution. For example, the first row of $H_{n}$ is $[1,1, \ldots, 1]$ and thus $S_{0}=\sum_{k=0}^{n-1} c_{k}$. The magnitude of $S_{0}$ is equal to $2 l$ if $n / 2+l$ $c_{k}$ 's are +1 and $n / 2-l c_{k}$ 's are -1 . The probability of this occurance can be readily evaluated. Similar considerations apply to other $S_{k}$ as well. Consequently, the distribution of the magnitude of $S_{k}(0 \leq k<n)$ is given by

$$
\operatorname{Pr}\left(\left|S_{k}\right|=2 l\right)=\left\{\begin{array}{cc}
\frac{1}{2^{n}}\left(\begin{array}{c}
n \\
n / 2
\end{array}\right) & l=0 \\
\frac{1}{2^{n-1}}\left(\begin{array}{c}
n \\
\frac{n+2 l}{2}
\end{array}\right) & 0<l \leq n / 2
\end{array}\right.
$$

The corresponding cumulative distribution function (cdf) of $\left|S_{k}\right|(0 \leq k<n)$ is a staircase function and is given by

$$
F_{1}(\zeta)=\left\{\begin{array}{cc}
\sum_{l=0}^{m} \operatorname{Pr}\left(\left|S_{k}\right|=2 l\right) & 2 m \leq \zeta<2 m+2 \\
1 & n<\zeta
\end{array}\right.
$$

The distribution of the PAPR relates to the distribution of $\max \left(\left|S_{0}\right|\left|S_{1}\right|, \ldots,\left|S_{n-1}\right|\right)$, which is simply the above raised to the power $n$ provided $S_{k}(0 \leq k<n)$ independently distributed. We assume that they are independent. As shown below using simulation results, this assumption leads to an accurate expression for the distribution. The $\mathrm{CCDF}$ of the PAPR is then given by

$$
F(\zeta)=1-\left[F_{1}(\zeta)\right]^{n}
$$

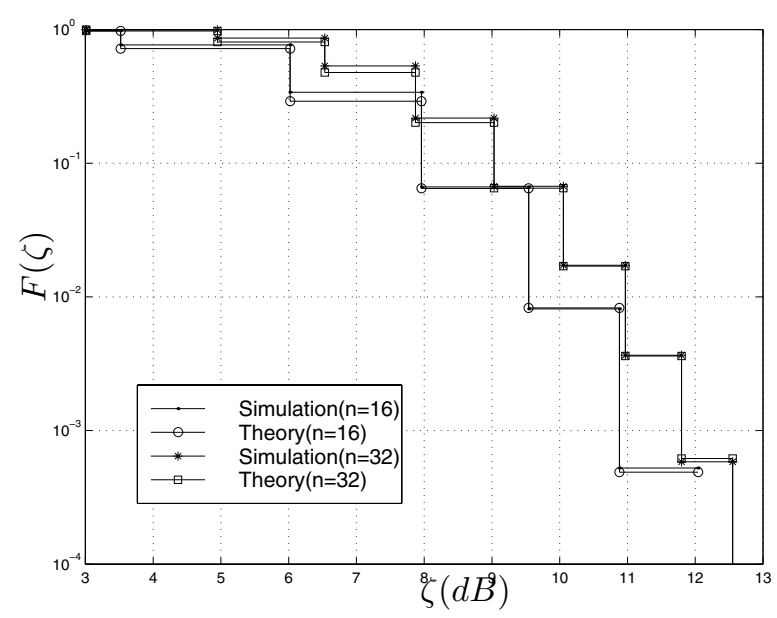

Fig. 2. CCDF of PAPR of Normal MC-CDMA System

Fig. 2 shows the CCDF of the PAPR as a function of $n$. For $n=32$, the PAPR exceeds $12.5 \mathrm{~dB}$ for only 1 out $10^{4}$ of all data blocks and for $90 \%$ of all data blocks, the PAPR is below $9 \mathrm{~dB}$. Moreover, the theoretical results for CCDF agree with simulation results.

The above CDF is an approximation, albeit a good one. In fact, some probabilities can be computed exactly. Paterson has shown that for any word c of length $n$ [18],

$$
\operatorname{PAPR}(\mathbf{c})=n\left(1-\frac{2 d_{*}(\mathbf{c})}{n}\right)^{2}
$$


where $d_{*}(\mathbf{c}):=\min \left\{d_{H}(\mathbf{c}, w): w \in \operatorname{RM}(1, m)\right\}$ denotes the minimum Hamming distance between $\mathbf{c}$ and the first-order Reed-Muller(RM) code of length $2^{m}$.

For each $\operatorname{RM}(1, m)$ codeword, we can find $\left(\begin{array}{l}n \\ l\end{array}\right)$ binary sequences at a Hamming distance $l$ from it. Provided $l \leq 2^{m-2}$, two sequences at distance $l$ from two distinct $\operatorname{RM}(1, m)$ codewords cannot be the same. Based on this observation, we have

$\operatorname{Pr}\left\{\operatorname{PAPR}(\mathbf{c})=\frac{(n-2 l)^{2}}{n}\right\}=\left(\begin{array}{l}n \\ l\end{array}\right) \frac{2^{m+1}}{2^{n}}$ for $0 \leq l \leq 2^{m-2}$

This exact probabilities may also be used to evaluate upper tails of the PAPR distribution.

\section{Achievable coding rate}

Consider block coding to reduce the PAPR to $\zeta$. This can in principle be achieved by excluding all sequences with PAPR over $\zeta$. From (7), the achievable coding rate is

$$
R(\zeta)=\frac{\log _{2}\left[2^{n} \operatorname{Pr}(\operatorname{PAPR}(\mathbf{c}) \leq \zeta)\right]}{n}=1-\log _{2}\left[F_{1}(\zeta)\right] .
$$

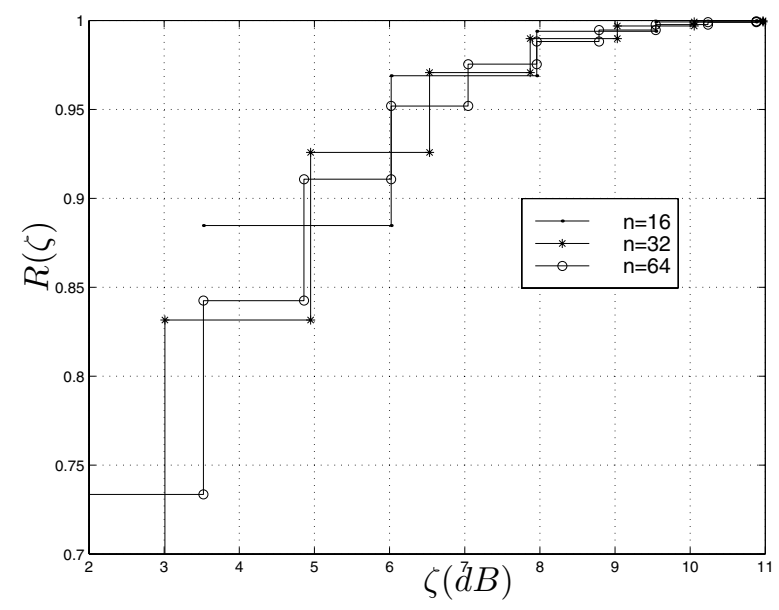

Fig. 3. Variation of Code Rate with PAPR

Fig. 3 shows the variation of code rate as a function of PAPR. For $n=32$, PAPR can be reduced below $3 \mathrm{~dB}$ with a code rate of 0.7. However, finding low PAPR codes are difficult for large $n$.

\section{Reducing PAPR USING MULtiPle Signal GENERATION}

The basic idea behind this approach is to generate multiple, independent symbols to represent an input data frame and select the minimum PAPR symbol for transmission. There are several techniques based on this idea and these primarily differ in the way they generate the multiple symbols. Another issue with this approach is the need for side information to tell the receiver which one of the signals has been used. Suppose $M \geq 1$ 'independent' MC-CDMA symbols are generated for an information sequence. The $\mathrm{CCDF}$ of the minimum of these is given by

$$
\operatorname{Pr}\left(\mathrm{PAPR}_{\min } \geq \zeta\right)=\left[F_{c}(\zeta)\right]^{M} .
$$

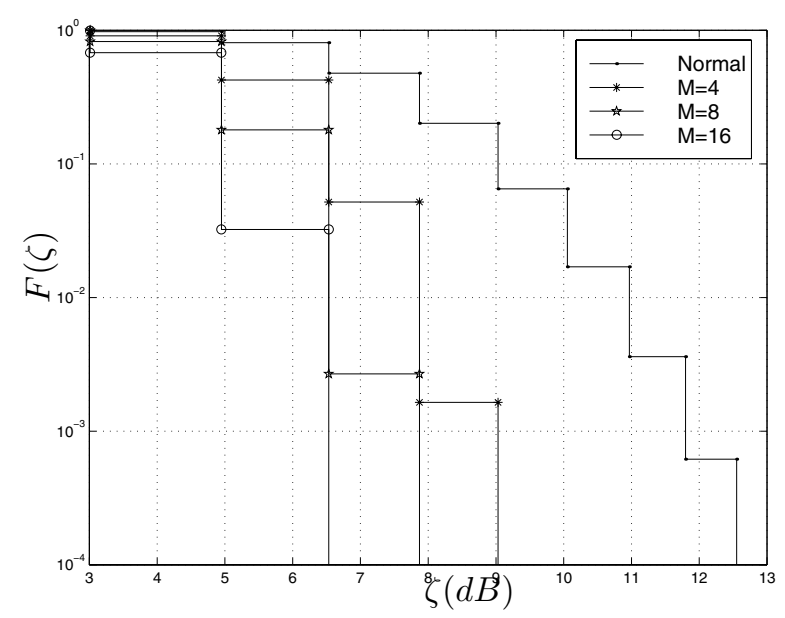

Fig. 4. CCDF of PAPR of Multiple Signal Generated MC-CDMA System for $n=64$

Fig. 4 shows the CCDF of the multiple signal generation method as a function of $M$ for $n=32$. Both PAPR reduction and complexity increase with $M$.

\section{A. Partial Transmit Sequences (PTS)}

In PTS, the input data block is partitioned into disjoint subblocks or clusters which are combined to minimize the peaks. We partition the data block $\mathbf{c}$ into $M$ disjoint subblocks, represented by the vectors $\left\{\mathbf{c}_{m}, m=1,2, \ldots, M\right\}$, such that $\mathbf{c}=\left[\mathbf{c}_{1}, \mathbf{c}_{2}, . ., \mathbf{c}_{M}\right]$. It is assumed that each subblock consists of a contiguous set of data symbols and the subblocks are of equal size. Then, each subblock is multiplied by a weighting factor $b_{m}(m=1,2, \ldots, M)$. PAPR can be reduced by optimizing the weighting factor $\mathbf{b}=\left[b_{1}, b_{2}, \ldots, b_{M}\right]$. Finally, the optimal PAPR can be found as

$$
\operatorname{PAPR}_{\text {optimal }}=\frac{1}{n} \min _{b_{1}, \ldots, b_{M}}\left[\max \left|H_{n}\left(\sum_{m=1}^{M} \mathbf{c}_{m} b_{m}\right)^{T}\right|^{2}\right] .
$$

For simplicity, we select $b_{m} \in\{+1,-1\}$. Moreover, the receiver must know the multiplexed sequence $\mathbf{b}$ to recover the data. Thus, a pointer to the phase factors must then be transmitted to the receiver as side information [20,21]. This requires at least $\log _{2} M$ bits to be transmitted as side information. If these bits are embedded in the OFDM data block, the loss in efficiency is $\frac{\log _{2} M}{n}$. This is negligible for large $n$. However, with differential modulation schemes, side information need not be transmitted. 


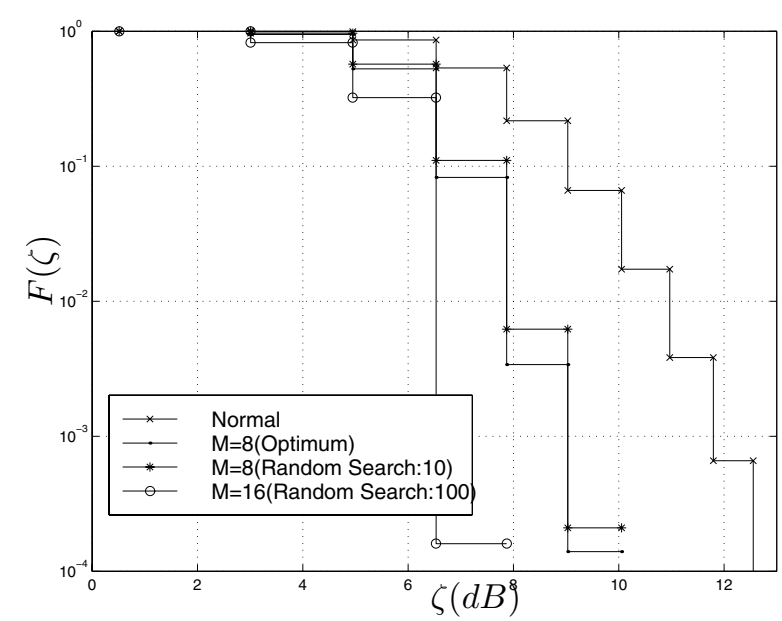

Fig. 5. CCDF of PAPR of PTS MC-CDMA System with $n=64$

Fig. 5 shows the CCDF of PTS for $n=32$. For $M=8$, the PAPR exceeds $10 \mathrm{~dB}$ for only 1 out $10^{4}$ of all data blocks whereas that of normal case is for only 1 out 10 . There is about a $2.5 \mathrm{~dB}$ reduction in PAPR in PTS with $M=8$ over a normal system. This causes a negligible code rate loss of 0.05 . This agrees with the results in Fig. 3 , where PAPR can be reduced below $10 \mathrm{~dB}$ with almost unity code rate.

Fig. 5 also shows how the performance varies with $M$. When $M$ is large, the PAPR reduction is large. However, the computational complexity also increases with $M$ (the optimized phase sequence requires $2^{M-1}$ computations of PAPR). Thus, performance can be traded off against complexity. For $M \geq 8$, the full search of all possible $\mathbf{b}$ is not implemented here. As suggested in [21], we randomly generate a number of phase sequences and choose the best in reducing PAPR.

\section{B. Selected Mapping (SLM)}

$M$ statistically independent alternative transmit sequences $\mathbf{a}^{(m)}$ represent the same information. The sequence with lowest PAPR is selected for transmission. To generate $\mathbf{a}^{(m)}$, we define $M$ distinct fixed vectors $\mathbf{b}^{(m)}=$ $\left[b_{0}^{(m)}, \ldots, b_{n-1}^{(m)}\right]$ with $b_{k}^{(m)} \in\{+1,-1\} 0 \leq k<n, 1 \leq m \leq$ $M$. Then, each data symbol $\mathbf{c}=\left[c_{0}, c_{1}, \ldots, c_{n-1}\right]$ is multiplied symbol-wise with the $M$ vectors $\mathbf{b}^{(m)}$, resulting in a set of $M$ different data symbols $\mathbf{c}^{(m)}$ with components

$$
c_{k}^{(m)}=c_{k} \times b_{k}^{(m)} \text { for } 0 \leq k<n \text { and } 1 \leq m \leq M .
$$

Finally, the optimal PAPR can be found as

$$
\operatorname{PAPR}_{\text {optimal }}=\frac{1}{n} \min _{\mathbf{b}^{(1)}, \ldots, \mathbf{b}^{(M)}}\left[\max \left|H_{n}\left(\mathbf{c}^{(m)}\right)^{T}\right|^{2}\right] .
$$

In order to recover the data, a pointer to the multiplied sequence has to be transmitted to the receiver as side information [20].

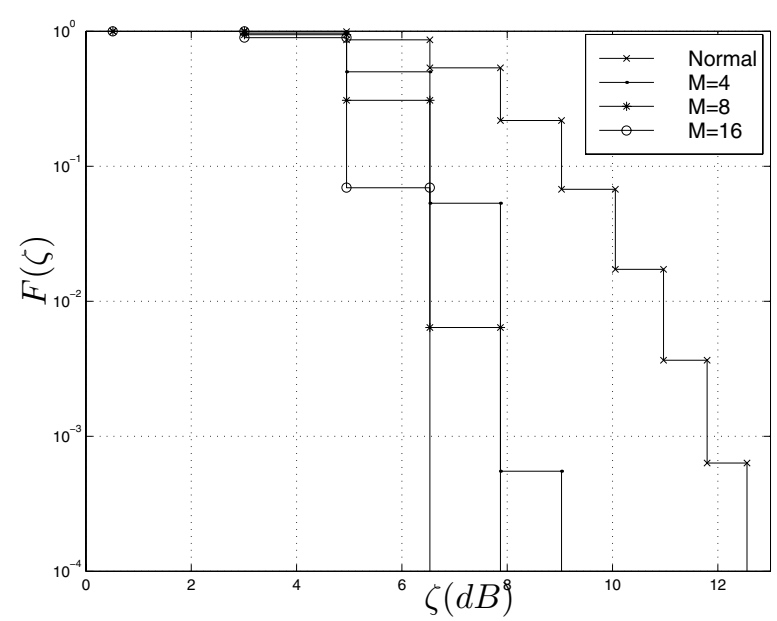

Fig. 6. CCDF of PAPR of SLM MC-CDMA System with $n=64$

Fig. 6 shows the CCDF of the PAPR as a function of $M$ for $n=32$. For $M=4$, only 1 out $10^{4}$ of data frame exceeds a PAPR of $10 \mathrm{~dB}$ whereas in the normal case, 1 out 10 of data frame exceeds a PAPR of $9 \mathrm{~dB}$. That amounts to a $3.5 \mathrm{~dB}$ reduction in PAPR, which is better than that of the PTS approach. However, both the PAPR reduction and computational complexity increase with $M$. Consequently, as with PTS, performance can be traded off against complexity.

\section{Impairments in the Presence of NONLINEARITIES}

The MC-CDMA signal is passed through a High Power Amplifier (HPA) to obtain a sufficient power level required for transmission. As mentioned previously, the HPA introduces nonlinear distortion because of operating at or close to saturation region. This causes out-of-band radiation and Bit Error Rate (BER) degradation. However, out-of-band radiation is not a significant problem since the MC-CDMA signal occupies a wider bandwidth. Thus, BER degradation is of interest in this nonlinear studies [12-17]. The nonlinear distortion of MC-CDMA signals increases, when amplified with nonlinear power amplifiers operating at lower back-offs. Thus, high PAPR of MC-CDMA signal requires high back-offs at the amplifiers, which is inefficient.

The non-linearity present in the amplifier can be expressed by its AM/AM component $F[\rho]$ and AM/PM component $\Phi[\rho]$, where $\rho$ is the magnitude of the signal and $\mathrm{AM}$ and PM represent amplitude modulation and phase modulation respectively. The input output relationship of solid-state power amplifiers (SSPA) can be modelled as

$$
\begin{aligned}
& F[\rho]=\frac{\rho}{\left[1+\left(\frac{\rho}{A}\right)^{2 \beta}\right]^{\frac{1}{2 \beta}}} \\
& \Phi[\rho]=0
\end{aligned}
$$

where the parameter $\beta$ controls the smoothness of the tran- 
sition from the linear region to the limiting or saturation region. These models satisfy the non-expansive property and give a maximum output signal of $A$. The input backoffs (IBO) of an amplifier can be expressed as

$$
\mathrm{IBO}=10 \log _{10}\left\{\frac{A^{2}}{E\left\{|x|^{2}\right\}}\right\}
$$

where $E\left\{|x|^{2}\right\}$ is the average of the amplifier input power.

\section{A. Simulation Results}

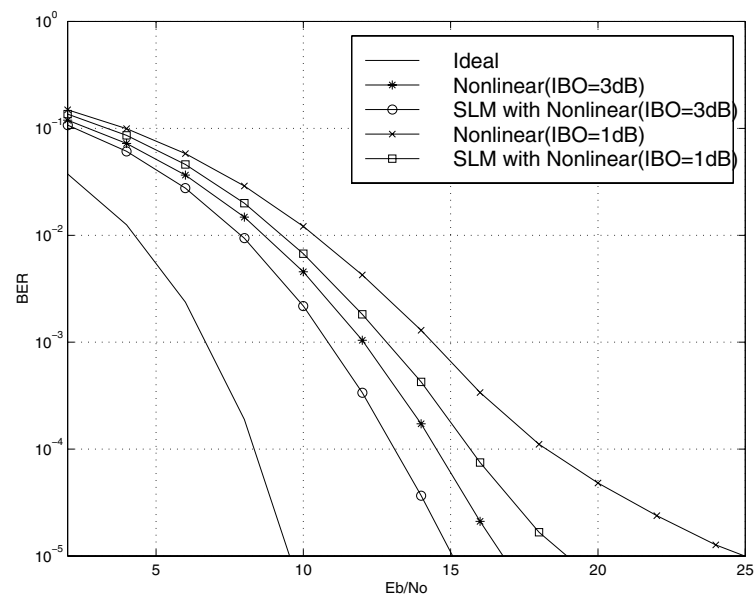

Fig. 7. BER of MC-CDMA signal passed through SSPA

Fig. 7 shows the BER of the MC-CDMA signal subject to SSPA nonlinearities. We assume coherent symbol detection in an AWGN channel and perfect SI at the receiver. With an input back-off of $3 \mathrm{~dB}$, SLM offers an SNR gain of $2 \mathrm{~dB}$ at $10^{-5}$ BER. When the back-off of the amplifier is decreased, BER degradation is increased and resulting in error floor. However, SLM reduces this error floor and offers an SNR gain of $6 \mathrm{~dB}$ at $10^{-5}$ BER with input back-off of $1 \mathrm{~dB}$.

We have used SLM to show that PAPR reduction will translate into BER improvement. Similar BER performance improvement can be obtained for PTS as well.

\section{Conclusion}

This paper has analyzed the PAPR problem in MCCDMA systems. Theoretical expressions for the CCDF of PAPR and achievable code rate are derived. PTS and SLM methods have been used to reduce PAPR. They improve the PAPR statistics of the MC-CDMA signal at the expense of additional complexity, but with little loss in data rate. For $n=32$ and $M=8$, PTS reduces PAPR by $2.5 \mathrm{~dB}$ whereas that of SLM is $4.5 \mathrm{~dB}$ at the clipping level of $10^{-4}$. We have also shown the BER performance improvement by SLM. SLM reduces the error floor caused by amplifier nonlinearities and offers an SNR gain of $6 \mathrm{~dB}$ at $10^{-5}$ BER with an input back-off of $1 \mathrm{~dB}$.

\section{REFERENCES}

[1] F. Adachi, M. Sawahashi, and H. Suda, "Wideband DSCDMA for next-gneration mobile communications systems," IEEE Commun. Mag., vol. 36, pp. 56-69, Sept. 1998.

[2] M. Zeng, A. Annamalai and V. K. Bhargava, "Harmonization of global third generation mobile systems," IEEE Commun. Mag., vol. 38, pp. 94-104, Dec. 2000.

[3] M. Zeng, A. Annamalai and V. K. Bhargava, "Recent advances in cellular wireless communications," IEEE Commun. Mag., vol. 37, pp. 128-138, Sept. 1999.

[4] C.-L. I and R. D. Gitlin, "Multi-code CDMA wireless personal communications networks," in IEEE ICC, pp. 1060-1064, IEEE, 1995.

[5] A. E. Jones, T. A. Wilkinson and S. K. Barton, "Block coding scheme for reduction of peak to mean envelope power ratio of multicarrier transmission schemes," IEE Elect. Lett., vol. 30, pp. 2098-2099, 1994.

[6] A. E. Jones and T. A. Wilkinson, "Minimisation of the peak to mean envelope power ratio of multicarrier transmission schemes by block coding," in IEEE Vehicular Technology Conference, vol. 2, pp. 825-829, IEEE, 1995.

[7] J. A. Davis and J. Jedwab, "Peak-to-mean power control in OFDM, Golay complementary sequences, and Reed-Muller codes," IEEE Trans. Inform. Theory., vol. 45, pp. 2397-2417, Nov. 1999.

[8] K. G. Paterson, "Generalized Reed-Muller codes and power control in OFDM modulation," IEEE Trans. Inform. Theory., vol. 46, pp. 104-120, Jan. 2000.

[9] T. Ottosson, "Precoding for minimization of envelope variations in multicode DS-CDMA systems," Wireless Personal Communications, vol. 13, pp. 57-58, May 2000.

[10] T. Ottosson, "Precoding in multicode DS-CDMA systems," in Int. Symp. Info. Thy., (Ulm, Garmany), p. 351, IEEE, June 1997.

[11] T. Ottosson and T. Palenius, "The impact of using multicode transmission in the WCDMA system," in IEEE Vehicular Technology Conference, pp. 1550-1554, IEEE, 1999.

[12] S. I. Kim, G. Y. Jung, S. Y. Yoon, and H. S. Lee, "Enhancement of constant amplitude coding for multicode wideband CDMA systems," IEICE Trans. Commun., vol. E83-B, pp. 2550-2555, Nov. 2000.

[13] T. Wada, "Charatecristic of bit sequence applicable to constant amplitude orthogonal multicode systems," IEICE Trans. Fundamentals, vol. E83-A, pp. 2160-2164, Nov. 2000.

[14] T. Wada, T. Yamazato, M. Katayama, and A. Ogawa, "Error correcting capability of constant amplitude coding for orthogonal multi-code CDMA systems," IEICE Trans. Fundamentals, vol. E81-A, pp. 2166-2169, Oct. 1998.

[15] T. Wada, T. Yamazato, M. Katayama, and A. Ogawa, "A constant amplitude coding for orthogonal multi-code CDMA systems," IEICE Trans. Fundamentals, vol. E80-A, pp. 2477-2484, Dec. 1997.

[16] N. Guo and L. B. Milstein, "Uplink performance evaluation of multicode DS-CDMA systems in the presence of nonlinear distortions," IEEE J. Select. Areas. Commun., vol. 18, pp. 14181428, Aug. 2000.

[17] N. Guo and L. B. Milstein, "The impact of nonlinear amplification on multi-code CDMA systems," in IEEE ICC, pp. 10341038, IEEE, 2000.

[18] K. G. Paterson, "On codes with low peak-to-average power ratio for Multi-Code CDMA," Tech. Rep. HPL-2001-115, HP Laboratories, Bristol, UK, 2001

[19] K. Paterson, "Sequences for OFDM and Multi-Code CDMA: Two problems in algebraic coding theory," Tech. Rep. HPL2001-146, HP Laboratories, Bristol, UK, 2001.

[20] S. H. Müller, R. W. Bäuml, R. F. H. Fisher and J. B. Huber, "OFDM with reduced peak-to-average power ratio by multiple signal representation," Annals of Telecommunications, vol. 52, pp. 58-67, Feb. 1997.

[21] L. J. Cimini, Jr. and N. R. Sollenberger, "Peak-to-average power ratio reduction of an OFDM signal using partial transmit sequences," IEEE Commun. Lett., vol. 4, pp. 86-88, Mar. 2000. 